\title{
Angioscopic Observation of Acute Femoral Artery Occlusion Complicated by Use of Angio-seal Vascular Closure Device
}

\author{
Ryo Munakata, MD, ${ }^{1}$ Masamichi Takano, MD, ${ }^{* 1}$ Tomohito Hada, MD, ${ }^{1}$ Tetsuro Shimura, MD,${ }^{1}$ \\ Osamu Kurihara, MD, ${ }^{1}$ Nakahisa Kimata, MD,${ }^{1}$ Toru Inami, MD, ${ }^{1}$ Daisuke Murakami, MD, ${ }^{1}$ \\ Takayoshi Ohba, MD, ${ }^{1}$ Yoshihiko Seino, MD, ${ }^{1}$ Wataru Shimizu, MD, ${ }^{2}$ Kyoichi Mizuno, MD $^{3}$ \\ ${ }^{1}$ Cardiovascular Center, Nippon Medical School Chiba Hokusoh Hospital, Chiba, Japan \\ ${ }^{2}$ Department of Cardiovascular Medicine, Nippon Medical School, Tokyo, Japan \\ ${ }^{3}$ Mitsukoshi Health and Welfare Foundation, Tokyo, Japan
}

\begin{abstract}
Femoral arteries are the most popular vessels of vascular access for angiography or percutaneous catheter intervention because of their diameter and accessibility. After the catheter procedures, hemostasis at the puncture site is achieved with conventional manual compression or by use of several vascular closure devices (VCDs) in recent years. However, the use of these devices infrequently results in lower limb ischemia as their major complications. We highlight a case of acute femoral artery occlusion caused by Angio-Seal TM hemostatic device and angioscopic findings of the case. Angioscopy after revascularization by balloon angioplasty revealed a residual red and white thrombi and an inorganic pale mass protruding into the lumen. The color of the mass was considered strange in vivo. On the basis of angioscopic findings, it was speculated that collagen sponge of the Angio-Seal accidently penetrated in to the arterial wall and the collagen component in the lumen caused thrombotic occlusion. Interventional cardiologist should be aware that serious problems sometimes occur when VCDs are used.
\end{abstract}

Key words: angioscopy, acute thrombotic occlusion, complication, vascular closure device

\section{Introduction}

Use of vascular closure devices (VCDs) is now a standard method for achieving femoral artery hemostasis following catheter procedures of coronary and peripheral vascular disease angiography. Angio-Seal (St Jude Medical, St Paul, MN, US) arterial closure device is widely used for preventing bleeding from puncture site and facilitating early ambulation after the procedures. Nevertheless, this hemostatic device may invite unique complications, such as stenosis, occlusion, or peripheral embolism. This case report presents acute femoral artery occlusion after using Angio-Seal VCD. We observed the occluded segment by angioscopy and described potential causes of acute occlusion based on angioscopic findings.

\footnotetext{
* 1715, Kamagari, Inzai, Chiba 270-1694, Japan

e-mail: takanom@nms.ac.jp

(Received 2014.10.27; Accepted 2015.01.05)

doi.org/10.15791/angioscopy.cr.15.0006
}

\section{A Case Report}

A 74-year-old female suspicious of arteriosclerosis obliterans underwent angiography using a 6-Fr sheath in diameter via left femoral artery, so-called cross-over approach. Subsequently catheter intervention for stenotic lesions in the right common femoral and external iliac artery was successfully performed. An Angio-Seal device was used for vascular seal at the left femoral puncture site. The hemostasis with the Angio-Seal failed, and an additional manual compression became necessary for complete hemostasis. Immediately, after manual compression left popliteal artery and dorsalis pedis were well palpable. The day after the procedures, short distance intermittent claudication appeared and her ankle-brachial index decreased from a preoperative value of 0.85 to 0.61 . Physical examination showed absence of left popliteal and pedal pulses. An urgent aorto-femoral angiogram was performed through right femoral artery. Digital subtraction angiography of left limb showed total occlusion of 


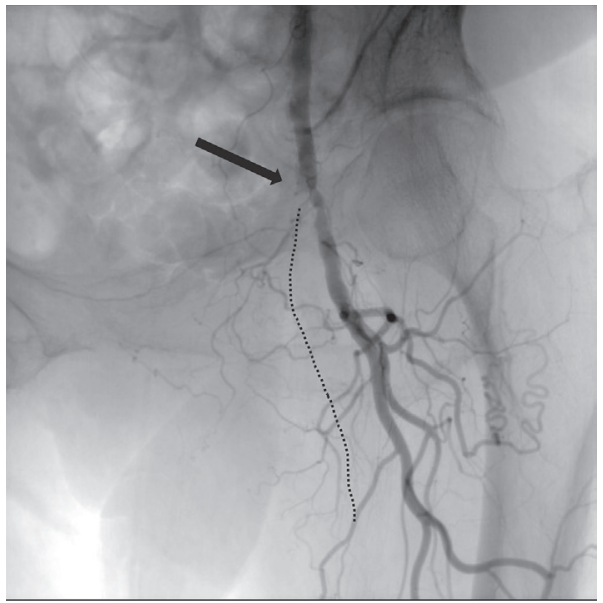

Fig. 1 Angiographic findings at the appearance of left limb ischemia

Angiography showed total occlusion in the left superficial femoral artery at the previous puncture site (arrow).

(A) Angioscopy
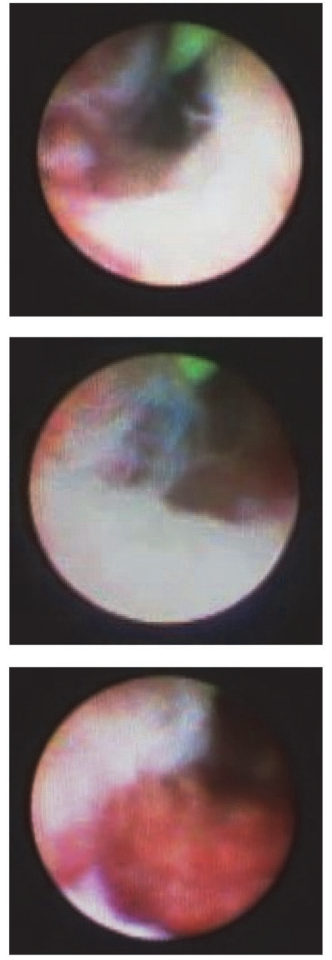

(B) Schema of angioscopy

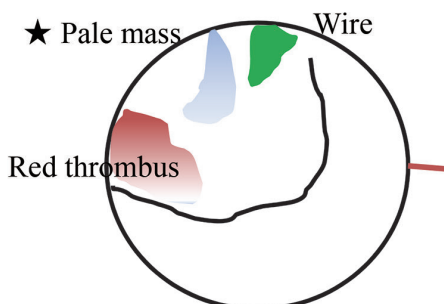

Wire

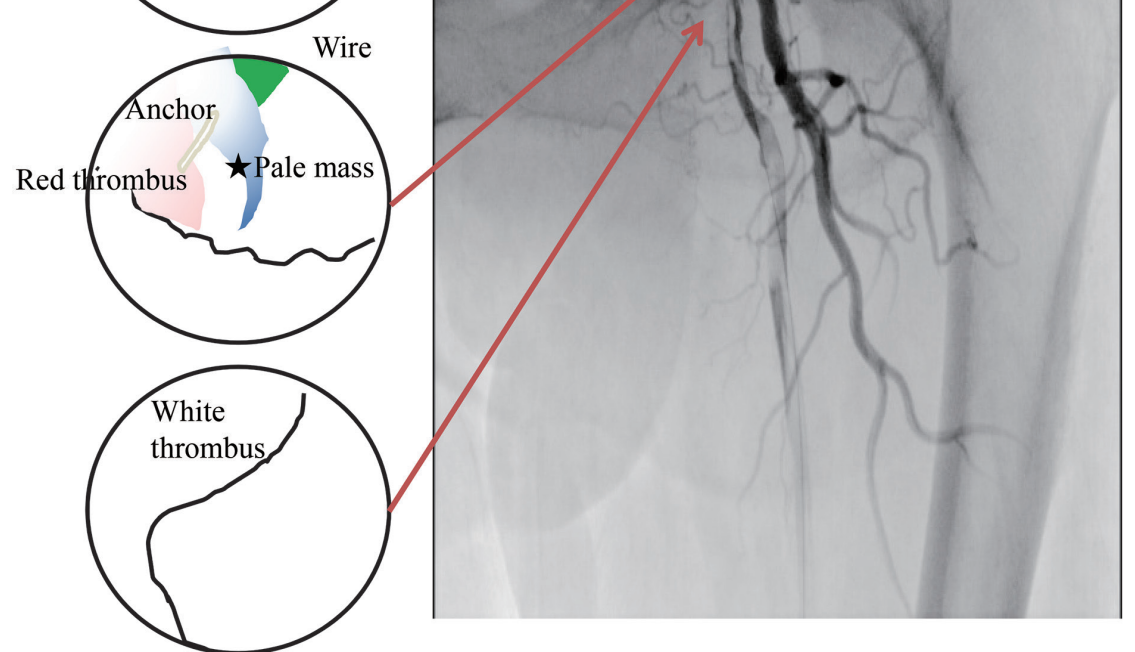

(C) Angiography

Fig. 2 Angioscopic and angiographic findings after balloon angioplasty

(A) Each part of angioscopic image. The upper section: proximal to the puncture site, there were protruding pale mass and red thrombi. The middle section: puncture site, the angioscopic image showed protruding pale mass, white linear structure thought to be anchor, and red thrombi. The lower section: distal of the puncture site. White thrombus is shown at the distal site. (B) Schema of angioscopic images. $\star$ : inorganic mass with pale color. (C) Angiography after the balloon angioplasty showed puncture site stenosis, filling defects, and linear dissection.

the superficial femoral artery (SFA) (Fig. 1) and developed collateral flow from deep femoral artery. Peripheral artery occlusion due to distal embolization was not found. Catheter intervention of the SFA was consequently attempted to recanalize the occluded vessel. Antegrade flow was obtained after dilatation of SFA with balloon angioplasty. After the recanalization, angios- copy with FULLVIEW NEO (FiberTech Co, Chiba, Japan) was performed to observe the occluded lumen. Angioscopy revealed a residual red and white thrombi and an inorganic pale structure. The structure protruded into the lumen and had strange color in the body of human beings (Fig. 2). Although angioplasty using scoring balloon (Angiosculpt TM, AngioScore, Fremont, CA, 
$5.0 / 20 \mathrm{~mm}$ ) at the maximum pressure of $8 \mathrm{~atm}$ was repeated, balloon indentation still remained. Angiography showed improvement of flow of the left limb. However, filling defects localized in the puncture site were left (Fig. 2). Therefore, thrombectomy with Fogarty catheter was attempted to remove the residual thrombus in the SFA. The collection of thrombectomy did not contain any piece of the Angio-Seal. Finally, saphenous vein patch and femoral-femoral bypass graft was performed because of residual left leg pain at rest, and the symptom completely disappeared.

\section{Discussion}

This is the first case that acute occlusion site originated from Angio-Seal VCD which was directly visualized by angioscopy. The Angio-Seal device has a fully absorbable anchor collagen sandwich mechanism and uses a ligature to tighten the collagen on to the arterial puncture site externally. The anchor keeps the collagen plug secured to the puncture site from inside of the vessel wall. According to manufacturer's recommendation, criterion for using the device safely permits its use only for common femoral artery punctures with an internal vessel diameter of 4 $\mathrm{mm}$ without focal plaque at or around the puncture site. However, in terms of vascular complications, it remains controversial as to whether VCDs are better than mechanical compression. ${ }^{1-3)}$ Goyen et al. reported that a complication rate of AngioSeal vascular closure was only $0.32 \%$ in a total of 6400 cases. $^{4)}$ At present, various vascular complications of arterial closure devices are known, such as hematoma, bleeding, arteriovenous fistula, pseudoaneurysm, arterial occlusion, and infection. ${ }^{3,5-7)}$ Bito $\mathrm{Y}$ et al. reports three cases of hemorrhagic and ischemic complications in acute phase related to Angio-Seal use. ${ }^{8)}$ The causes of their complication are considered as follows: (1) arteriosclerotic change at the puncture site, (2) vigorous tamping with inadequate tension, and (3) vascular atherosclerotic calcifications, except the puncture site, catching the anchor disk site. Stein et al. suggest that vigorous tamping with inadequate tension on the suture or deployment of the anchor too deep within the vessel can cause inappropriate placement of the collagen plug in the arterial lumen. ${ }^{9)}$ In this case, artificial material with pale color and thrombi was recognized by peripheral angioscopy. With regard to causes of acute occlusion, collagen sponge protruding into the lumen may provoke massive thrombus formation. There arised some technical problems about collage protrusion. First, operator may push Angio-Seal system strongly because latent atherosclerosis at the puncture site may disturb anchoring the system and sufficient hemostasis. Second, relatively lower puncture site and smaller diameter of the SFA may cause malposition of the anchor. VCDs such as Angio-Seal eliminate the need for prolonged arterial compression and reduce the subsequent interval of bed rest. However, local complications sometimes arise from the puncture site like the present case. To avoid the local vascular complication, the most important thing is to obtain the information of the puncture site and select a suitable case for VCDs before using them safely. In any cases, interventional cardiologist should be aware that serious problems sometimes occur when VCDs are utilized.

\section{Disclosure}

None of the authors have conflicts of interest to disclose.

\section{References}

1) Nikolsky E, Mehran R, Halkin A, et al: Vascular complications associated with arteriotomy closure devices in patients undergoing percutaneous coronary procedures: A meta-analysis. J Am Coll Cardio 2004; 44:1200-1209

2) Dregelid E, Jensen G, Daryapeyma A: Complications associated with the Angio-Seal arterial puncture closing device: Intra-arterial deployment and occlusion by dissected plaque. J Vasc Surg 2006; 44:1357-1359

3) Koreny M, Riedmuller E, Nikfardjam M, et al: Arterial puncture closing devices compared with standard manual compression after cardiac catheterization: Systematic review and meta-analysis. JAMA 2004; 291:350-357

4) Goyen M, Manz S, Kroger K, et al: Interventional therapy of vascular complications caused by the hemostatic puncture closure device angio-seal. Catheter Cardiovasc Interv 2000; 49:142-147

5) Jang JJ, Kim M, Gray B, et al: Claudication secondary to Perclose use after percutaneous procedures. Catheter Cardiovasc Interv 2006; 67:687-695

6) Thalhammer C, Aschwanden M, Jeanneret C, et al: Symptomatic vascular complications after vascular closure device use following diagnostic and interventional catheterization. Vasa 2004; 33:78-81

7) Andreotti F, Lavorgna A, Coluzzi G, et al: Lost and found: an unusual late complication of the Angio-Seal closure device. Int J Cardiol 2007; 117:e1-3

8) Bito Y, Sakaki M, Inoue K, et al: Surgical treatment of complications associated with the angio-seal vascular closure device: report of three cases. Ann Vasc Dis 2010; 3:144-147

9) Stein BC, Teirstein PS: Nonsurgical removal of angio-seal device after intra-arterial deposition of collagen plug. Catheter Cardiovasc Interv 2000; 50:340-342 